\title{
Electrohydrodynamic linear stability analysis of dielectric liquids subjected to unipolar injection in a rectangular enclosure with rigid sidewalls
}

\author{
A. T. Pérez ${ }^{1} \dagger$, P. A. Vázquez ${ }^{2}$ \\ Jian $\mathbf{W u}^{3}$ and P. Traoré ${ }^{3}$ \\ ${ }^{1}$ Departamento de Electrónica y Electromagnetismo, Universidad de Sevilla, Facultad de \\ Física, Avenida Reina Mercedes s/n, 41012 Sevilla, Spain \\ ${ }^{2}$ Departamento de Física Aplicada III, Universidad de Sevilla, ESI, Camino de los \\ Descubrimientos s/n, 41092 Sevilla, Spain \\ ${ }^{3}$ Institut PPRIME, Département Fluide-Thermique-Combustion, Boulevard Pierre et Marie \\ Curie, BP 30179, 86962 Futuroscope-Chasseneuil, France
}

(Received ?; revised ?; accepted ?. - To be entered by editorial office)

We investigate the linear stability threshold of a dielectric liquid subjected to unipolar injection in a 2D rectangular enclosure with rigid boundaries. A finite element formulation transforms the set of linear partial differential equations that governs the system into a set of algebraic equations. The resulting system poses an eigenvalue problem. We calculate the linear stability threshold, as well as the velocity field and charge density distribution, as a function of the aspect ratio of the domain. The stability parameter as a function of the aspect ratio describes paths of symmetry-breaking bifurcation. The symmetry properties of the different linear modes determine whether these paths cross each other or not. The resulting structure has important consequences in the non-linear behavior of the system after the bifurcation points.

Key words: Electrohydrodynamics, Unipolar injection, Linear instability, Finite element methods, Symmetry-breaking bifurcation

\section{Introduction}

Electrohydrodynamics(EHD) is an interdisciplinary field dealing with the interaction of fluids and electrostatics. It has important applications in several industrial processes (Melcher 1981; Crowley 1986). Some examples are EHD pumps (Seyed-Yagoobi 2005; Pearson \& Seyed-Yagoobi 2009; Ryu et al. 2010), heat transfer enhancement (Jones 1979; McCluskey \& Atten 1988; Seyed-Yagoobi \& Bryan 1999), EHD turbulent mixing (Jalaal et al. 2013), flow control (McCluskey \& Atten 1988; Traoré \& Louste 2011), and bio MEMS and nanotechnology applications (Darabi et al. 2001; Castellanos et al. 2003; Wong et al. 2004).

One of the most classical problems studied in EHD is the electroconvection in a infinite dielectric liquid sheet sandwiched between two parallel plates and subjected to an unipolar injection of ions (J C Lacroix \& Hopfinger 1975; Castellanos 1991, 1998). When an electric voltage is applied between the electrodes, the electric field pushes the free

$\dagger$ Email address for correspondence: alberto@us.es 
charges that appear due to the chemical reaction at the interface between liquids and electrodes. Above a certain voltage threshold the liquid is put into motion because of the loss of the stability. The fluid entrains the charges, while the distribution of charges determines the electric force and the motion of the liquid. Hence, a very strong non-linear coupling appears between the velocity field and the charge density distribution. The linear stability problem was studied by (Schneider \& Watson 1970) and (Atten \& Moreau 1972), and the non-linear stability was analyzed by (Atten \& Lacroix 1979).

The complexity of EHD convection has prompted to use the direct numerical techniques. However, the problem is also challenging from a numerical point of view. There are three mechanisms of charge transport: convection by the velocity of the liquid, drift by the electric field and diffusion. The last one is negligible when comparing to the other two, at least in the bulk (Castellanos 1998). Thus, the charge conservation equation becomes hyperbolic. Consequently, strong gradients of charge density may appear and the problem is very sensitive to the effects of numerical diffusion. Several numerical techniques have been used to tackle this difficulty: Particle-In-Cell (Castellanos et al. 1987; Chicón et al. 1997; Vázquez et al. 2006), Flux Corrected Transport (FCT) schemes (Pérez \& Castellanos 1989; Vázquez et al. 2006, 2008), Total Variation Diminishing (TVD) schemes (Traore \& Perez 2012), (Wu et al. 2013a) and, more recently, Discontinuous Galerkin finite element (Vázquez \& Castellanos 2013).

In order to numerically study the electoconvection in an infinite layer, the system is modelled as a finite layer with symmetric or periodic boundary conditions on the lateral walls. In the linear stability analysis, the stability criterion is determined as a function of the aspect ratio of the domain. Since an infinite system can choose any wavelength, the minimum of these threshold values is the linear stability criterion, and the corresponding width equals half the wavelength of the most unstable mode. Comparatively few works have dealt with the electroconvection in a finite enclosed cavity. Only a few experimental studies have been made, and most of them were concerned with cavities of large aspect ratios (Atten et al. 1980) or focused on the time dependent and chaotic behavior (Malraison \& Atten 1982).

In the free walls case, the bifurcation that defines the stability threshold is subcritical. Once the liquid is put into motion, the velocity of the liquid is finite, and greater than the ionic drift velocity. This finite velocity induces nonlinear effects of paramount importance. The main features of this "nonlinear" convective motion were deduced from a simple hydraulic model by (Felici 1969). There exist two criteria, linear and nonlinear, which are associated with a hysteresis loop and at which discontinuities occur in the steadystate current and velocity amplitude. The roll pattern of the flow originates a void region containing no electric charge. It is the existence of this void region and its boundary what makes this problem so sensitive to numerical diffusion.

In a recent paper, the authors studied numerically the electroconvection in an insulating liquid in a 2D enclosed cavity (Wu et al. 2013b). They observed that when no-slip boundary conditions are imposed on all the boundaries the nature of the bifurcation changes, and a supercritical branch appears for cavities with small aspect ratio. In this case, it is possible to find steady states of motion where the maximum velocity of the liquid is smaller than the ionic drift velocity. This bifurcation is similar to the one observed in Rayleygh-Bénard-Marangoni problems (van de Vooren \& Dukstra 1989). As the value of the stability parameter is further increased, a subcritical bifurcation arises similar to the one observed in an infinite system or the free walls case.

In this paper we study the linear stability of a dielectric liquid subjected to unipolar injection of ions enclosed in a 2D rigid container. We use the standard linear technique: the steady state is perturbed and a set of linear equations is obtained for the perturbed 
quantities. In the infinite case the perturbations can be expanded in Fourier modes, reducing the partial differential equations to a set of ordinary differential equations. However, when no-slip boundary conditions for the velocity are imposed, the problem is no longer separable and the whole set of partial differential equations must be solved. To this end we use a finite element method. The eigenvalues and eigenfunctions of the matrix built from the weak formulation of finite element approximate the eigenvalues and eigenfunctions of the continuous problem (Boffi 2010).

The paper is organized as follows. In the next section we state the physical domain, the governing equations, boundary conditions of both free and rigid walls cases, and the relevant non-dimensional parameters. Section 3 is devoted to the linear stability analysis. We develop the perturbations equations and define the corresponding eigenvalue problem. Then we apply the finite element technique and describe the structure of the matrices whose eigenvalues approximate those of the continuum problem. In section 4 we present the solutions of the eigenvalue problem. We use the free walls case as a test, since the results can be compared with other validated solutions. Then we describe the solutions for the rigid walls case. Here, we are able to reproduce the linear stability criterion obtained with the full numerical simulation ( Wu et al. 2013b). We analyze the structure of the eigenvalues and their eigenfunctions as the aspect ratio of the domain changes. Section 5 discusses the symmetry properties of the linear modes and their consequences for the bifurcation behavior of the systems. Finally, in the last section we summarize the results.

\section{Problem formulation}

We consider a perfectly insulating liquid confined in a 2D enclosure of height $d$ and width $L_{x}$. The top and bottom plates are electrodes. The liquid is assumed to be incompressible, Newtonian, and isothermal; with mass density $\rho$, kinematic viscosity $\nu$ and absolute permittivity $\varepsilon$. An electric potential $\Phi_{a}$ is applied to the bottom plate while the top electrode is grounded. We make the assumption that only the bottom electrode injects charges and only one type of charge carriers is present (unipolar injection). This can be achieved experimentally in different ways, for example, covering the electrode with a special membrane that injects ions into the liquid (J C Lacroix \& Hopfinger 1975). We will further assume that the density of injected charge on the bottom electrode, $q_{i}$ is constant (autonomous injection). The top electrode is assumed to behave as an open boundary for the ions. These are common assumptions in EHD problems and allow to understand a wide range of phenomena (Castellanos 1991).

The governing equations is a set of coupled partial differential equations including the Navier-Stokes equations, the charge density transport equation and Gauss' law for the electric field (Chicón et al. 1997). These equations can be written in dimensionless form using the scales $d, \Phi_{a}, \varepsilon \Phi_{a} / d^{2}$ and $K \Phi_{a} / d$ for, respectively, length, potential, charge 


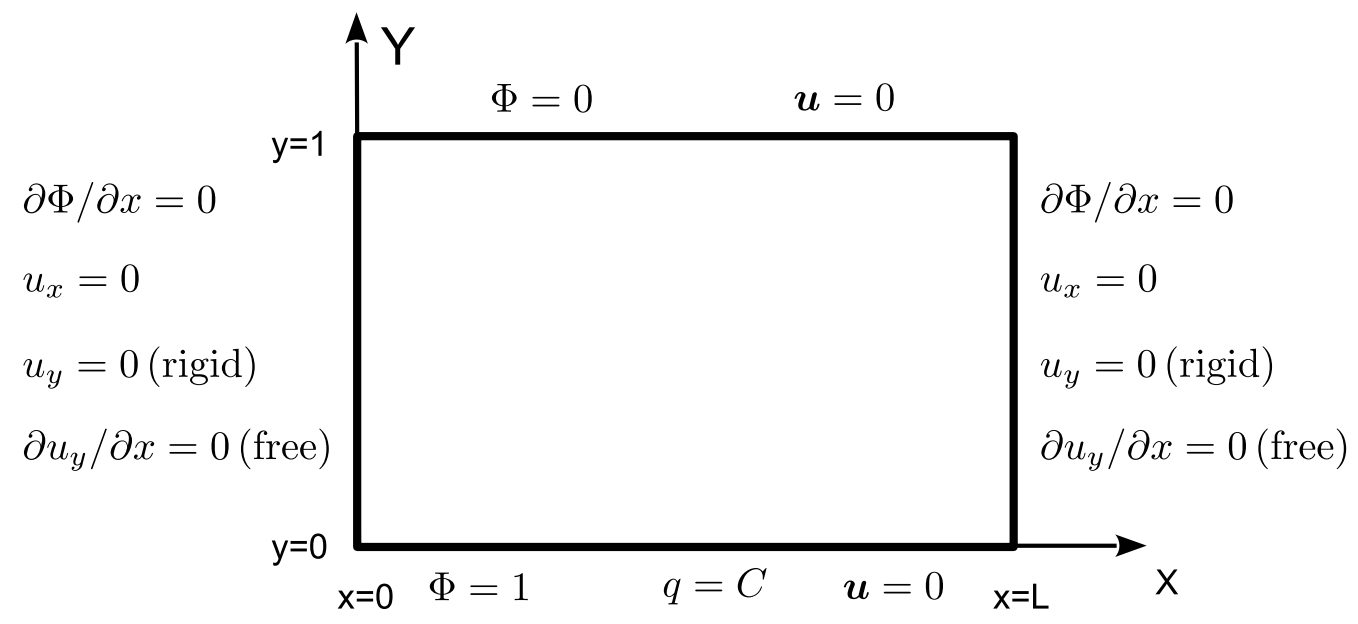

FIGURE 1. Non-dimensional computational domain with boundary conditions.

density and velocity. Here, $K$ stands for the ionic mobility. Thus we have

$$
\begin{aligned}
& \Delta \Phi=-q, \\
& \boldsymbol{E}=-\boldsymbol{\nabla} \Phi, \\
& \frac{\partial q}{\partial t}+\boldsymbol{\nabla} \cdot(q(\boldsymbol{u}+\boldsymbol{E}))=0, \\
& \boldsymbol{\nabla} \cdot \boldsymbol{u}=0, \\
& \frac{\partial \boldsymbol{u}}{\partial t}+(\boldsymbol{u} \cdot \boldsymbol{\nabla}) \boldsymbol{u}=-M^{2} \boldsymbol{\nabla} p+\frac{M^{2}}{T} \Delta \boldsymbol{u}+M^{2} q \boldsymbol{E} .
\end{aligned}
$$

The non-dimensional boundary conditions are

$$
\begin{array}{lllr}
y=0: & \phi=1, & u_{x}, u_{y}=0, & q=C \\
y=1: & \phi=0 & u_{x}, u_{y}=0, & \\
x=0, L: & \partial \phi / \partial x=0 & u_{x}, u_{y}=0 & \text { (rigid walls) } \\
& & u_{x}, \partial u_{y} / \partial x=0 & \text { (open walls) }
\end{array}
$$

Figure 1 shows the geometry and boundary conditions of the problem.

The non-dimensional parameters appearing in the equations and boundary conditions are

$$
T=\frac{\varepsilon \Phi_{a}}{\rho \nu K}, \quad C=\frac{q_{i} d^{2}}{\varepsilon \Phi_{a}}, \quad M=\frac{1}{K}\left(\frac{\varepsilon}{\rho}\right)^{1 / 2}, \quad L=\frac{L_{x}}{d} .
$$

The parameter $T$ is the ratio between the electric force, which reduces to the Coulomb force in this problem, and viscous terms in the Navier-Stokes equations. It is referred to as the electric Rayleigh number and will serve as the stability parameter. The injection number $C$ measures the injection strength. The mobility number $M$ is the ratio between the so called hydrodynamic mobility and the ionic mobility (Felici 1969). It is a property of the fluid under consideration. Finally, $L$ is the non-dimensional width of the box. 


\section{Linear stability analysis}

For small values of the applied voltage the viscosity prevents liquid motion. As the voltage is increased and exceeds a certain threshold, the liquid is eventually put into motion. This threshold can be computed from the linear stability analysis. The linear stability analysis provides the growth rate of small perturbations of the hydrostatic state. If all perturbations decay in time, the system is stable. The smallest value of the stability parameter for which some perturbations grow in time is the linear stability criterion $T_{c}$, the threshold above which the liquid motion appears.

Equations 2.1 admit a steady hydrostatic solution $\boldsymbol{u}_{0}=\mathbf{0}$. The hydrostatic electric field $\boldsymbol{E}_{0}=E_{0}(y) \boldsymbol{e}_{y}$, and the charge profile $Q_{0}(y)$ are subjected to the conditions:

$$
Q_{0}(y=0)=C, \quad \int_{0}^{1} E_{0}(y) \mathrm{d} y=1
$$

The solutions for the electric field and the charge density are:

$$
E_{0}=E_{s} \sqrt{1+2 C y / E_{s}}, \quad Q_{0}=\frac{C}{\sqrt{1+2 C y / E_{s}}}
$$

whre $E_{s}$ is the value of $E_{0}$ at the injector, and it is obtained from (3.1).

We look for perturbations of the basic state. Specifically, any variable in the system is written as its value in the hydrostatic state plus a perturbation:

$$
\begin{aligned}
& q(x, y, t)=Q_{0}(y)+q^{\prime}(x, y, t) \\
& \Phi(x, y, t)=\Phi_{0}(y)+\Phi^{\prime}(x, y, t) \\
& \boldsymbol{E}(x, y, t)=\boldsymbol{E}_{0}(y)+\boldsymbol{E}^{\prime}(x, y, t) \\
& p(x, y, t)=P_{0}(y)+p^{\prime}(x, y, t) \\
& \boldsymbol{u}(x, y, t)=\boldsymbol{u}^{\prime}(x, y, t)
\end{aligned}
$$

The primed quantities are supposed to be much smaller than the basic state quantities. Introducing these expressions in (2.1) and keeping only the first order terms in the perturbations, one obtains:

$$
\begin{aligned}
& \Delta \Phi^{\prime}=-q^{\prime} \\
& \boldsymbol{E}^{\prime}=-\nabla \Phi^{\prime} \\
& \boldsymbol{\nabla} \cdot \boldsymbol{u}^{\prime}=0 \\
& \frac{\partial \boldsymbol{u}^{\prime}}{\partial t}=-M^{2} \nabla p^{\prime}+\frac{M^{2}}{T} \Delta \boldsymbol{u}^{\prime}+M^{2}\left(Q_{0} \boldsymbol{E}^{\prime}+q^{\prime} \boldsymbol{E}_{0}\right) \\
& \frac{\partial q^{\prime}}{\partial t}+\nabla \cdot\left(Q_{0}\left(\boldsymbol{u}+\boldsymbol{E}^{\prime}\right)+q^{\prime} \boldsymbol{E}_{0}\right)=0
\end{aligned}
$$

Since the equations are linear, the standard procedure is to seek for an exponential grow 
of the perturbations:

$$
\left(\begin{array}{c}
\boldsymbol{u}^{\prime} \\
p^{\prime} \\
\Phi^{\prime} \\
q^{\prime}
\end{array}\right)=\left(\begin{array}{c}
\boldsymbol{U}(x, y) \\
\Pi(x, y) \\
V(x, y) \\
Q(x, y)
\end{array}\right) \exp (\sigma t)
$$

The factor $\sigma$ is the growth rate and, in principle, it is a complex quantity. When $\operatorname{Im}(\sigma)=0$ it is said that the principle of exchange of stabilities applies. As it is discussed in (Atten \& Moreau 1972), it is not possible to prove rigorously that this principle applies in the free walls case. However, the numerical simulation conducted by (Traore \& Perez 2012) and (Wu et al. 2013a) shows that the growth of small perturbation proceeds in a monotonic way, without oscillations. And this behavior is observed for weak, moderate and strong injection, i. e. , independent of the value of $C$ used in the simulation. This is a clear indication that $\operatorname{Im}(\sigma)=0$, and, therefore, the principle of exchange of stabilities applies. Here, we will assume that $\operatorname{Im}(\sigma)=0$ both in the free and rigid walls situations. Then, in the marginal state, which corresponds to the onset of motion, it is $\sigma=0$. The comparison with the results issued from the numerical analysis validates this assumption.

For the free walls case, equations (3.4) admit an expansion in terms of normal modes with a separate $x$ and $y$ dependence. For example, it is possible to find a solution of the form:

$$
\left(\begin{array}{c}
\boldsymbol{u}^{\prime} \\
p^{\prime} \\
\Phi^{\prime} \\
q^{\prime}
\end{array}\right)=\left(\begin{array}{c}
\boldsymbol{U}(y) \\
\Pi(y) \\
V(y) \\
Q(y)
\end{array}\right) \exp (\sigma t) \exp \left(i k_{x} x\right)
$$

But, in general, the system is not separable. In particular, in the rigid walls case, the factorization (3.6) cannot fulfill simultaneously the boundary conditions for the charge and the velocity at the lateral walls. This forces us to use a more general method of solution.

Since we are interested in the marginal stability, $\sigma=0$, we drop the time derivative terms in equations (3.4). In this way, the number $M$ disappears and has no influence in the linear stability criterion. The pressure can be rescaled to include the parameter $T$ in its definition, i.e., we replace $p$ with $T p$. Also, we drop the primes from the magnitudes. Then the linear stability equations take the final form

$$
\begin{aligned}
& \boldsymbol{\nabla} \cdot \boldsymbol{u}=0 \\
& \boldsymbol{\nabla} p-\Delta \boldsymbol{u}=T\left(-Q_{0} \boldsymbol{\nabla} V+q \boldsymbol{E}_{0}\right) \\
& \boldsymbol{\nabla} \cdot\left(Q_{0}(\boldsymbol{u}-\boldsymbol{\nabla} V)+q \boldsymbol{E}_{0}\right)=0 \\
& \Delta V=-q
\end{aligned}
$$

to which we must add the corresponding boundary conditions:

$$
\begin{array}{lllc}
y=0: & V=0 & u_{x}=u_{y}=0 & q=0 \\
y=1: & V=0 & u_{x}=u_{y}=0 & \\
x=0, L: & \partial V / \partial x=0 & u_{x}=\partial u_{y} / \partial x=0 & \text { (free walls) } \\
& & u_{x}=u_{y}=0 & \text { (rigid walls) }
\end{array}
$$




\begin{tabular}{cc}
$L_{\min }$ & $T_{\min }$ \\
\hline 0.614 & 164.09 \\
1.230 & 164.09 \\
1.843 & 164.09 \\
2.457 & 164.09
\end{tabular}

TABLE 1. Values of $T$ for the minimums in figure 2 .

Equations (3.7) along with boundary conditions (3.8) define an eigenvalue problem. The secular determinant leads to a relation between the relevant parameters of the form

$$
\mathcal{F}(T, C, L)=0 .
$$

We consider $T$ as the stability parameter. For every set of values of the parameters $C$ and $L$, equation (3.9) provides a set of values of $T$ each one corresponding to a different linear mode. The smallest of these eigenvalues $T_{c}$ is the linear stability threshold.

The eigenvalue problem can be approached using a finite element formulation (Boffi 2010). We choose Continuous Galerkin finite element as trial and tests functions. The orders of the finite element spaces are 2 for the velocity, 1 for the pressure, 2 for the electric potential and 2 for the charge density. Introducing the test and trial functions, the resulting algebraic problem takes the form

$$
A x=T B x
$$

where $x$ is a vector built with the unknown values of the variables at the nodes of the mesh, and the matrices $A$ and $B$ come from the integrals of the weak formulation of equations (3.7). This is a generalized eigenvalue problem (Cliffe et al. 1994). The eigenvalues of (3.10) approximates the eigenvalues of the continuous problem. The generalized eigenvalue problem (3.10) is solved using DOLFIN (Logg \& Wells 2010), an interface to the FEniCS package (Eds et al. 2010). This is a framework for automated solution of differential equations by the Finite Element method. The package allows the construction of the matrices $A$ and $B$ using a high level formulation of the weak problem. It also handles the computation of eigenvalues and eigenfunctions through the SLEPc package (Hernandez et al. 2005), a software library for the solution of large scale sparse eigenvalue problems.

For given values of the non-dimensional parameters $C$ and $L$, the solution of the eigenvalue problem posed by equation (3.10) provides the corresponding value of the parameter $T$ and the eigenvector $x$. This eigenvector contains the finite element approximation to the velocity, charge distribution, electric potential and pressure that corresponds to that value of $T$. For a given set of values of $L$ and $C$ several values of $T$ are possible, each one corresponding to different charge and velocity distributions.

\section{Results}

In this section we present the results for the case $C=10$, both with free and rigid walls. All the computations have been made with a regular mesh with $\Delta h=0.01$ in both directions. Hence, the number of nodes increases with the domain width $L$. 


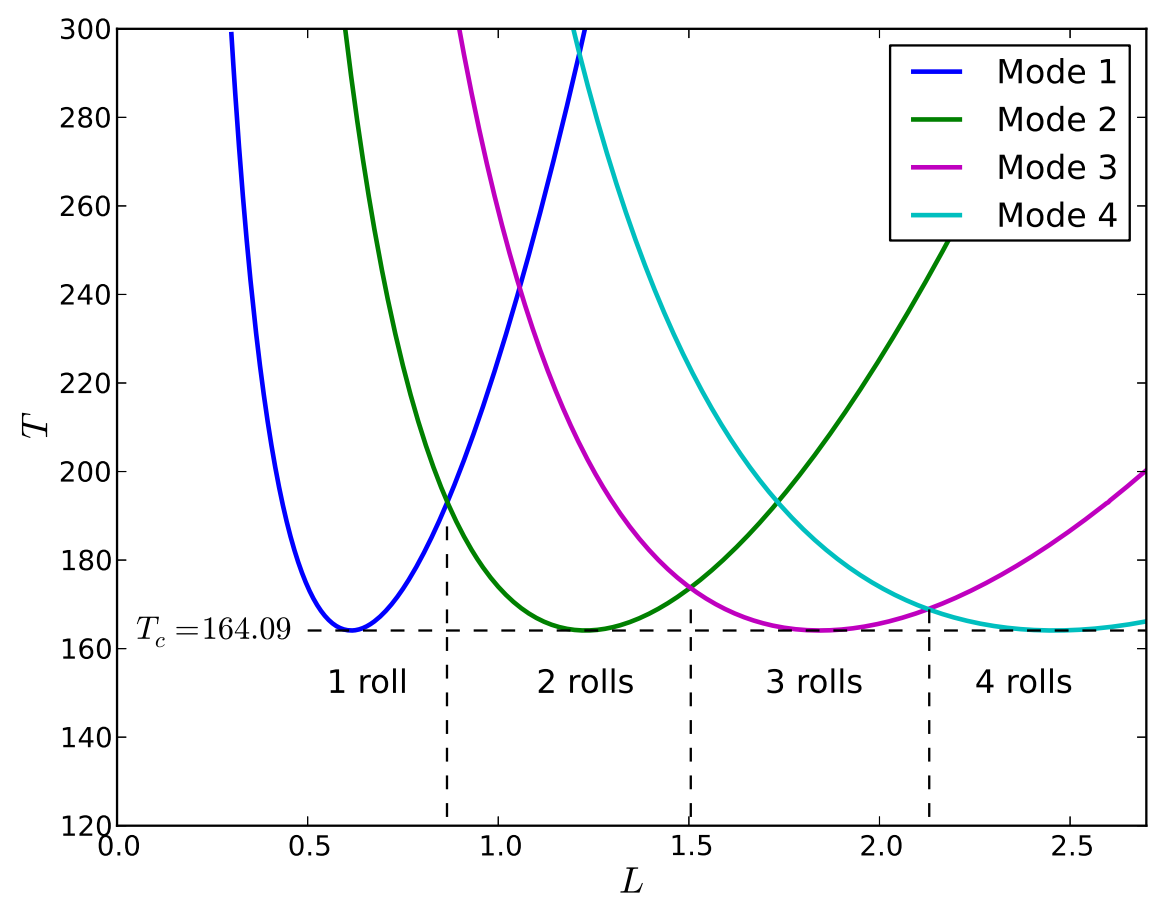

Figure 2. Eigenvalues of the first four modes as a function of $L$ for the free walls case $(C=10)$. The number of rolls of the most unstable modes and the linear stability criterion are indicated in the figure.

\subsection{Free walls}

As a validation of the overall procedure we have computed the stability diagrams for the free walls case. This case also corresponds to the linear stability analysis of a liquid layer of infinite horizontal extension, a case that was studied by (Schneider \& Watson 1970) and (Atten \& Moreau 1972). Figure 2 shows the eigenvalues of the first four modes as a function of the size of the domain. For a given value of $L$ each mode becomes unstable at a different value of $T$. The stability threshold is given by the smallest eigenvalue. For $L<0.870$ the mode with one roll is the most unstable one. As $L$ increases, the eigenvalue for the mode with two rolls becomes smaller than the eigenvalue of the one roll mode (for $L>0.870$ ) in the figure. As $L$ increases further, the modes with more rolls become the more unstable ones. In this figure and the next ones the computations have been performed with a step value of $\Delta L=0.01$.

The free walls case is a way to model an infinite domain. In an infinite domain, perturbations of all wavelengths are possible, and the basic state becomes unstable when the instability parameter reaches the value of the the smallest eigenvalue. This minimum is the threshold value of instability of the parameter $T$. The values of the minima and the corresponding values of $L$ are shown in table 1 . The value of $T_{\min }$ is the same for all the modes, up to the fifth digit. Also the domain widths for those minima are exact multiples of the width of the first mode. In fact, as figure 3 shows, the structure of the flow for the n-mode corresponds to $n$ single rolls juxtaposed, with the signs alternatively reversed. So the linear stability criterion for an infinite domain is $T_{c}=164.09$ and the 

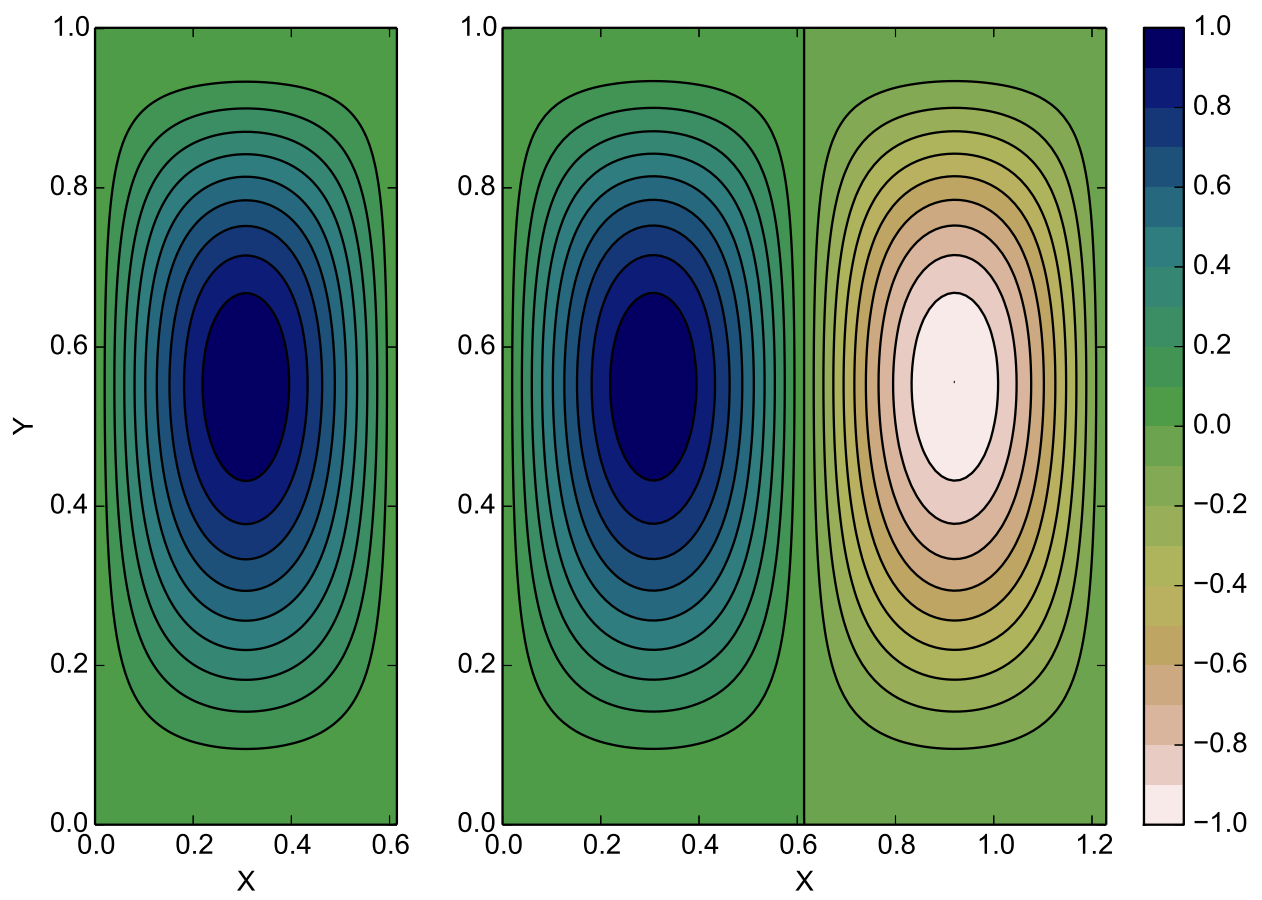

FiguRE 3. Stream function contour plots of the eigenfunctions of the most unstable modes for $L=0.614$ (left) and $L=1.228$ (right). The pattern of the two rolls case is a juxtaposition of two simple rolls (free walls case).

most unstable half wavelength is $L_{c}=0.614$. Atten and Moreau obtained $L_{\min }=0.614$ and $T_{\min }=164.1$ for the case of strong injection $(C=10)$ and rigid electrodes. This agreement validates our method.

\subsection{Rigid walls}

Figure 4 plots the smallest eigenvalue as a function of the width of the domain for the case of rigid walls. For each value of $L$, the smallest eigenvalue gives the linear stability criterion $T_{c}$. The figure also shows the values of $T_{c}$ computed in (Wu et al. $2013 b$ ) where the complete set of governing equations was solved using a finite volume technique. The agreement is excellent. We can observe some cusps in the graphic. The first one is highlighted in the inset. Each cusp marks the transition from a flow pattern to another with a different number of main rolls. The number of main rolls, once the flow is fully developed, in the flow structure for each range of $L$ is marked in the figure. Again, as $L$ increases the number of rolls of the most unstable mode increases. However, the structure is different to that of the free walls case (compare figure 4 to 2). The value of $T_{c}$ decreases as $L$ increases, except when approaching the cusps. There are relative minima between cusps, but the values of $T_{c}$ at these minima are smaller as the size of the domain increases. For large values of $L$, the value of $T_{c}$ tends to the threshold value computed for an infinite system, as the domain becomes more and more similar to an infinite domain: for $L=20$ we obtain $T_{c}=165$. Also the width of a convective cell tends to the value predicted for the infinite system. This is illustrated in figure 5 , which is a plot of the ratio between $L$ and the number of rolls as a function of $L$. The evolution takes the form 


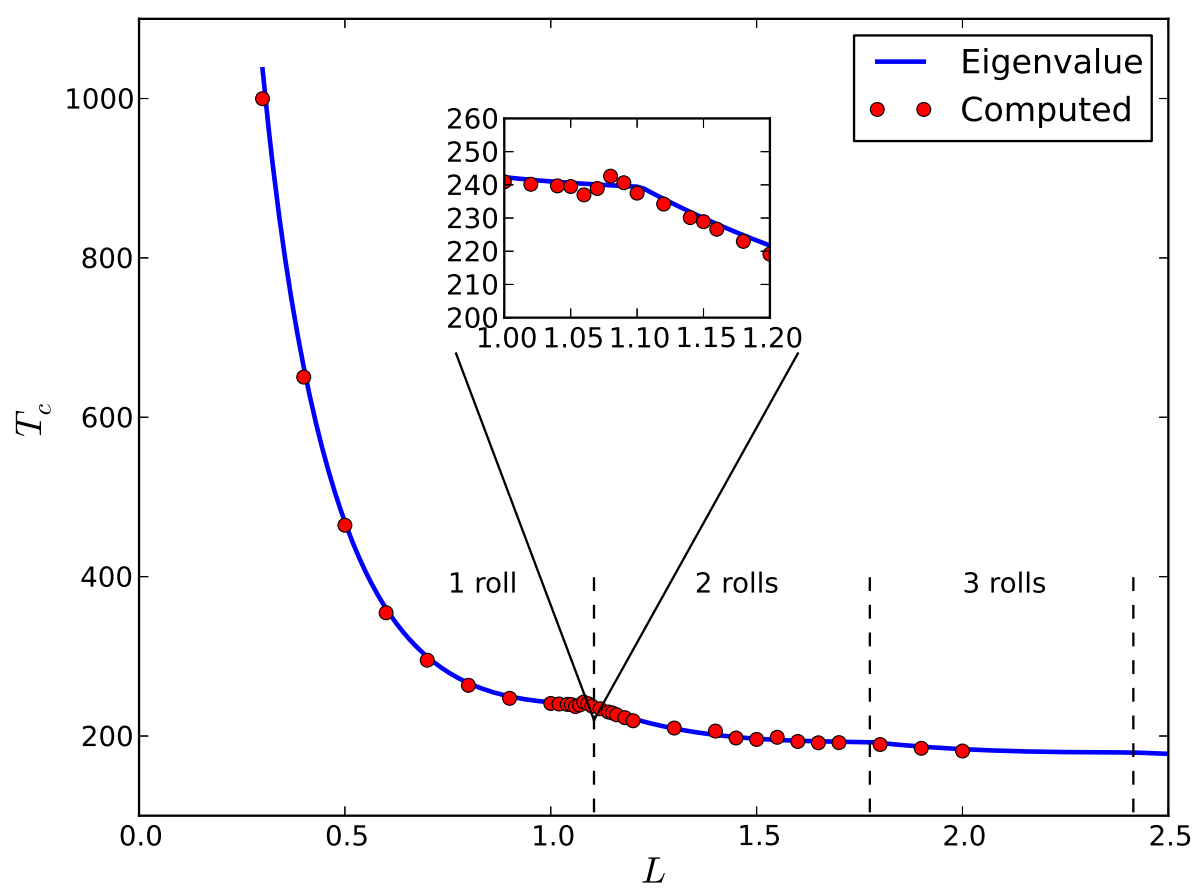

Figure 4. Eigenvalue of the most unstable mode as a function of $L$ for $C=10$ and rigid walls (solid line). The dots are the values obtained in (Wu et al. 2013b) from a numerical simulation of the whole set of equations.

of a saw-tooth modulation, because the width of every convective cell grows with $L$ until a new convective cell appears and the ratio diminishes abruptly.

Figure 6 plots the first four eigenvalues for the rigid walls case with $C=10$ as a function of the domain width $L$. We see that the cusps in figure 4 correspond to the values of $L$ where the two lower modes cross each other. If we compare figures 2 and 6 we see that the behavior of the modes is completely different in the free and rigid walls cases. In figure 2 all the modes intersect each other in the $L-T$ diagram. In figure 6 , when no-slip conditions apply on all the boundaries, modes 1 and 2 cross each other, as well as modes 3 and 4 . However modes 1 and 3, and modes 2 and 4 do not cross each other. The direct numerical simulation of the complete set of equations cannot reveal this underlying structure.

Figures 7 and 9 show the contour lines of the stream functions corresponding to modes 1 and 2 for three different values of $L$. The first value of $L$ has been chosen between the first crossing of modes 1 and 2 in the $L-T$ diagram (figure 6 ), the second between the first and second crossing, and the third one between the second and third crossing. Hence, mode 1 is the most unstable mode for $L=0.900$ and $L=2.200$ (Figures 7.A and 7.C), and mode 2 is the most unstable one for $L=1.600$ (Figure 9.B).

There is an important difference between these stream functions and those of the free walls case depicted in figure 3 . The no-slip condition on the lateral walls produces counter rolls at the four corners (figure 8). This is one of the reasons why figure $9 . \mathrm{B}$ cannot be built as a juxtaposition of two rolls of figure 7.A, since the two rolls of figure 9.B do 


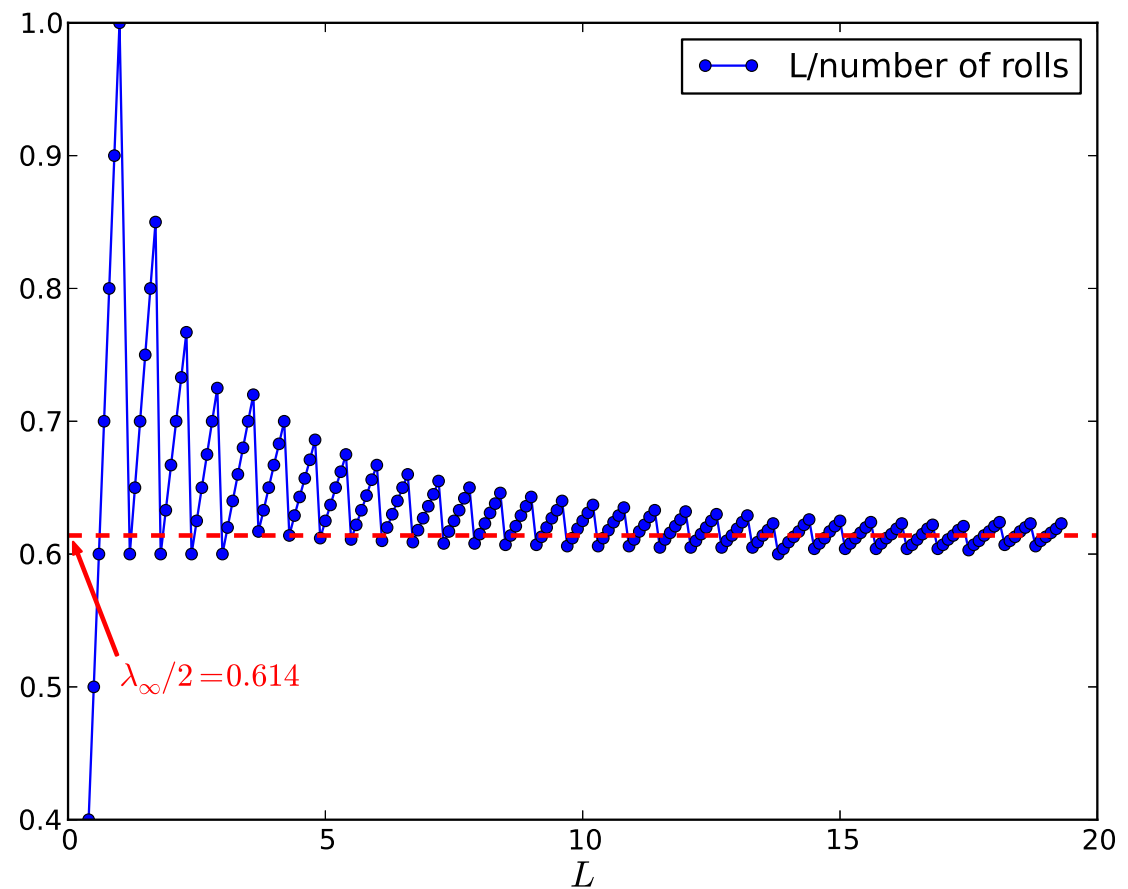

FiguRE 5. Width of a convective cell (computed as the ratio between the domain length $L$ and the number of rolls) as a function of $L$. The linear theory for the infinite case predicts a value of 0.614 .

not have counter rolls in the middle. The other reason is that the liquid velocity in the border between the rolls in figure 9.B is not zero.

Mode 1 has always an odd number of main rolls. When $L$ is small there is room only for one roll (Figure 7.A). As $L$ increases, the counter rolls at the corners grow and, eventually, the rolls of the same side merge into one roll, smaller than the main one (Figure 7.B). These lateral rolls grow with $L$ until the three rolls become of similar size (Figure 7.C). This process keeps on as $L$ grows, increasing the number of rolls. The behavior is similar for mode 2 , but with an even number of rolls.

In the free walls case it is possible to assign to every mode a number equal to the number of rolls of the corresponding velocity distribution. In the rigid walls case this is not possible, because the structure of the velocity field of every mode changes continuously when $L$ changes, producing different number of rolls for different values of $L$ for the same mode.

\section{Discussion}

\subsection{Stability and symmetry breaking}

The paths traced by the different modes in figure 6 are similar to those found in RayleighBénard or Marangoni convection (Cliffe \& Winters 1986; Winters et al. 1988), and are paths of bifurcation points. A similar behavior is encountered whenever certain paths of bifurcation points avoid crossing. The origin of this phenomenon is to be found in the symmetry characteristics of the different modes. 


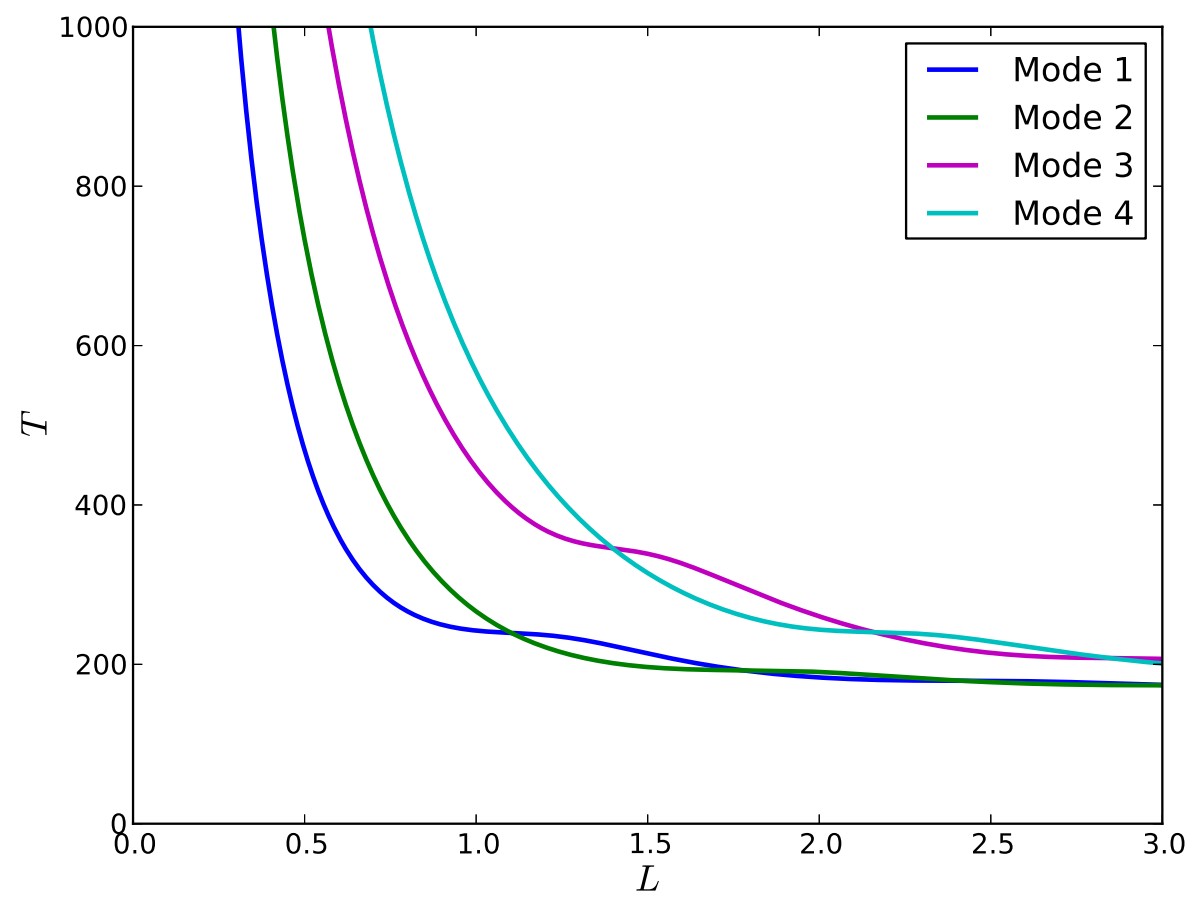

FiguRE 6. The first four eigenvalues, corresponding to four different modes, as a function of $L$ for $C=10$ and rigid walls.

The hydrodynamic basic state is symmetric with respect to reflections about the horizontal as well as the vertical mid-planes. The liquid motion breaks one or both symmetries. The parity of the different modes of liquid motion implies a different symmetry for odd and even modes. The stream functions of the even modes maintain the symmetry with respect to the vertical plane, whereas for the odd modes they loose both symmetries. Therefore, the bifurcations are symmetry-breaking. The even modes break one symmetry and the odd modes break two. Both types of modes, even or odd, represent a different class of symmetric solutions. For even modes (mode 2, 4, etc..) the stream function is an odd function of $x$ (with respect to the mid-plane of the domain), whereas the charge density perturbation is an even function of $x$ (with respect to the mid-plane of the domain). Conversely, for odd modes the stream function is even and the charge density perturbation is odd.

In general, in a two parameters system two paths of symmetry-breaking bifurcation points intersect only if they break different symmetries (or if the solutions belong to different symmetry classes), whereas the path of bifurcation points that break the same symmetry cannot cross one another (van de Vooren \& Dukstra 1989; Cliffe \& Winters 1986; Winters et al. 1988). A simplified argument may help to understand this fact. For every symmetry class it is possible to reduce the domain to a half of the complete domain, for example to $0 \leqslant x \leqslant L / 2$, being the functions for $L / 2 \leqslant x \leqslant L$ obtained applying the corresponding symmetry. Therefore, for every symmetry class there will be a different eigenvalue problem, because the boundary conditions at $x=L / 2$ will be different. The question to be asked now is when is it possible to have multiple eigenvalues of a given 

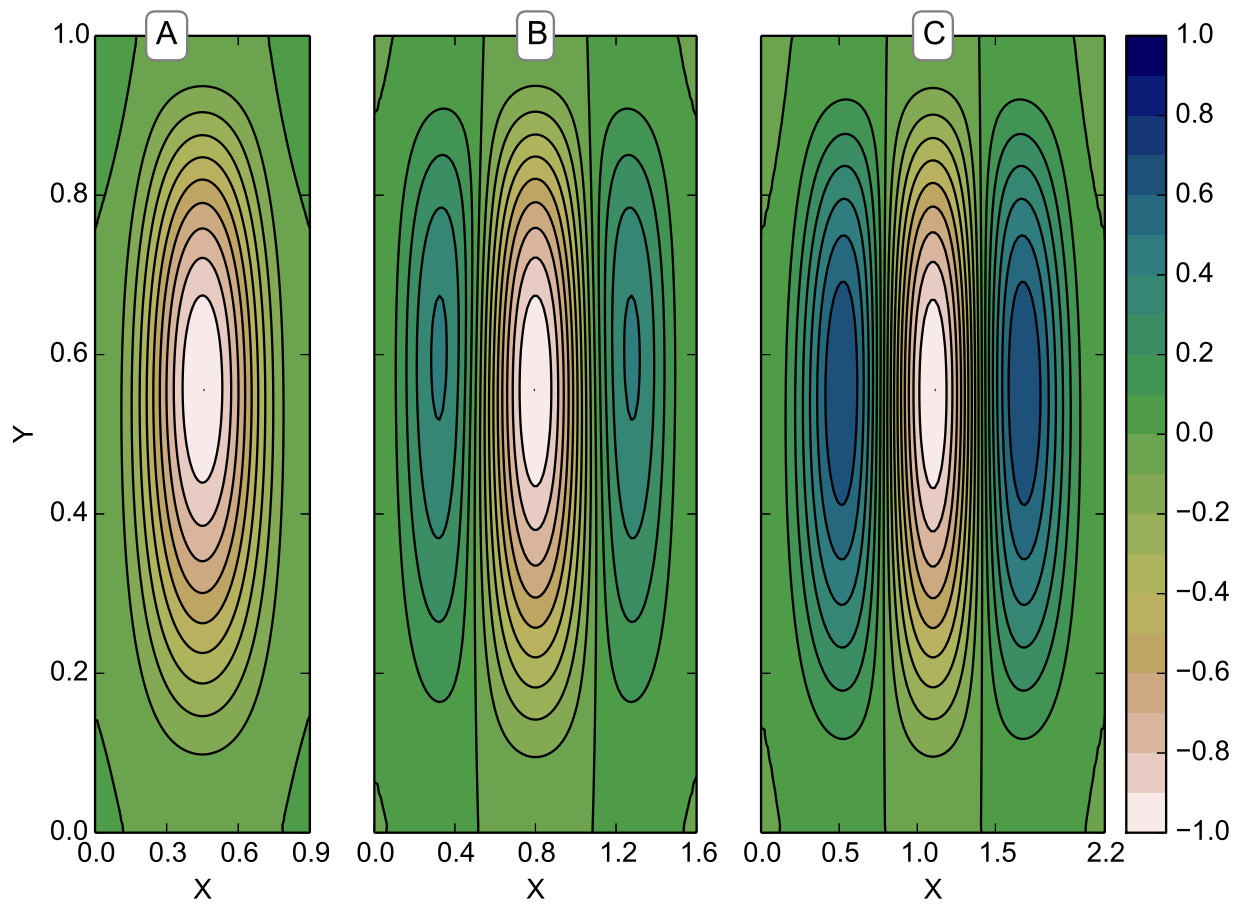

Figure 7. Stream function contour plots of the eigenfunction of mode 1 for $L=0.900,1.600,2.200$. This is the most unstable mode for the first and third value of $L$.

matrix. The following argument is taken from (van de Vooren \& Dukstra 1989). To fix ideas let us consider a $2 \times 2$ symmetric matrix:

$$
A=\left(\begin{array}{ll}
a_{11} & a_{12} \\
a_{12} & a_{12}
\end{array}\right)
$$

This matrix will have two equal eigenvalues (two paths will cross) if and oly if the discriminant for the characteristic function is zero:

$$
\left(a_{11}-a_{22}\right)^{2}+4 a_{12}^{2}=0
$$

This is possible only if both terms, $a_{11}-a_{22}$ and $a_{12}$, are zero simultaneously. If the matrix depends on parameters, the minimum number of parameters needed to satisfy both conditions, in a non-accidental way, is two. In our case, once we have fixed $C$, the problem depends only on the parameter $L$, being $T$ the eigenvalue. Therefore, for every value of $L$ there is only one mode per symmetry class with a given value of $T$. This is why the paths traced by modes 1 and 2 cross each other but the paths traced by modes 1 and 3 do not.

Concerning the case of free sidewalls it is seen that all curves can intersect each other. This is due to the fact that the solutions for the stream function and charge density can be written as the product of a function of $x$ and a function of $y$. This factorization reduces the problem to an eigenvalue problem in $y$ with an additional parameter $k_{x}$ (see equation (3.6)) and the eigenvalue problems for different values of $k_{x}$ are completely independent, 


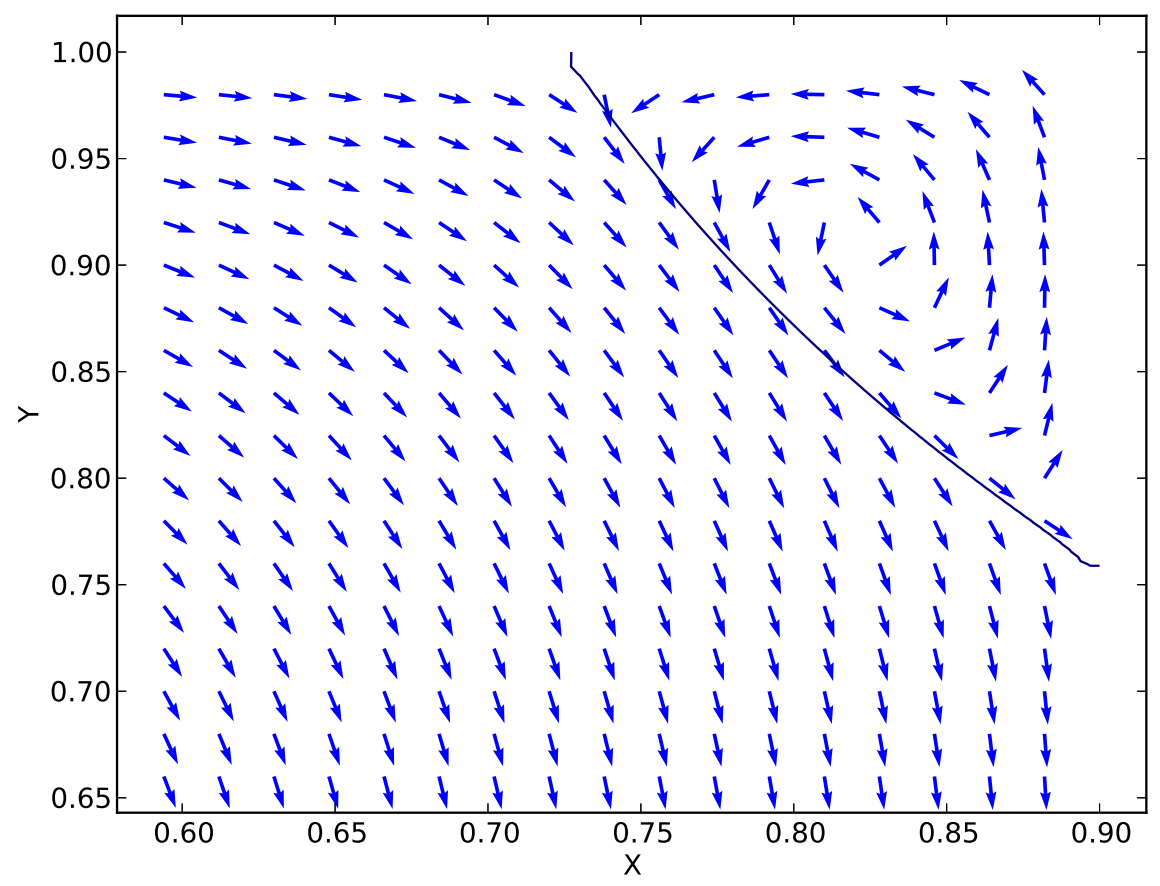

Figure 8. Detail of the velocity field of the eigenfunction of mode 1 for $L=0.900$. The plot reveals the presence of a counteroll near the corner.

and may have equal eigenvalues. The free walls case is in some way a degenerate case (see the discussion in (van de Vooren \& Dukstra 1989)).

\subsection{Relation between the linear stability analysis and the bifurcation diagrams}

As we said in the introduction, the bifurcation at the linear instability threshold is subcritical in the free walls case and may be supercritical in the rigid walls one. In (Wu et al. 2013b) we numerically solved the full set of equations and studied how the bifurcation changes when the aspect ratio of the domain is varied. Depending on the value of the aspect ratio supercritical or subcritical bifurcations may appear. For $L<1.05$, there is a first supercritical bifurcation followed by a subcritical one. There are two linear instability thresholds. The first one $T_{c 1}$ is associated to the transition from the rest state to motion with a velocity smaller than the drift velocity. The second one $T_{c 2}$ marks the transition to a motion characterize by a velocity greater than the drift velocity. For $L \sim 1.05$, the supercritical behavior disappears and $T_{c 1}$ and $T_{c 2}$ merge. As it is clear now, this point corresponds to the crossing of the first and second unstable modes in the linear stability analysis (see figure 6). For $L>1.15$, the supercritical behavior is reencountered, and, again the border between both behaviors is marked by the crossing of the two most unstable modes.

(Castellanos 1991) proposed an analytical method to decide whether the bifurcation in EHD electroconvection is subcritical or supercritical in the case of weak injection. In this method a functional form of the liquid velocity is given and an equation for the evolution of the amplitude of the velocity field is obtained from the Navier-Stokes equation. It can be shown that the use of a single mode to describe the liquid velocity always leads to 

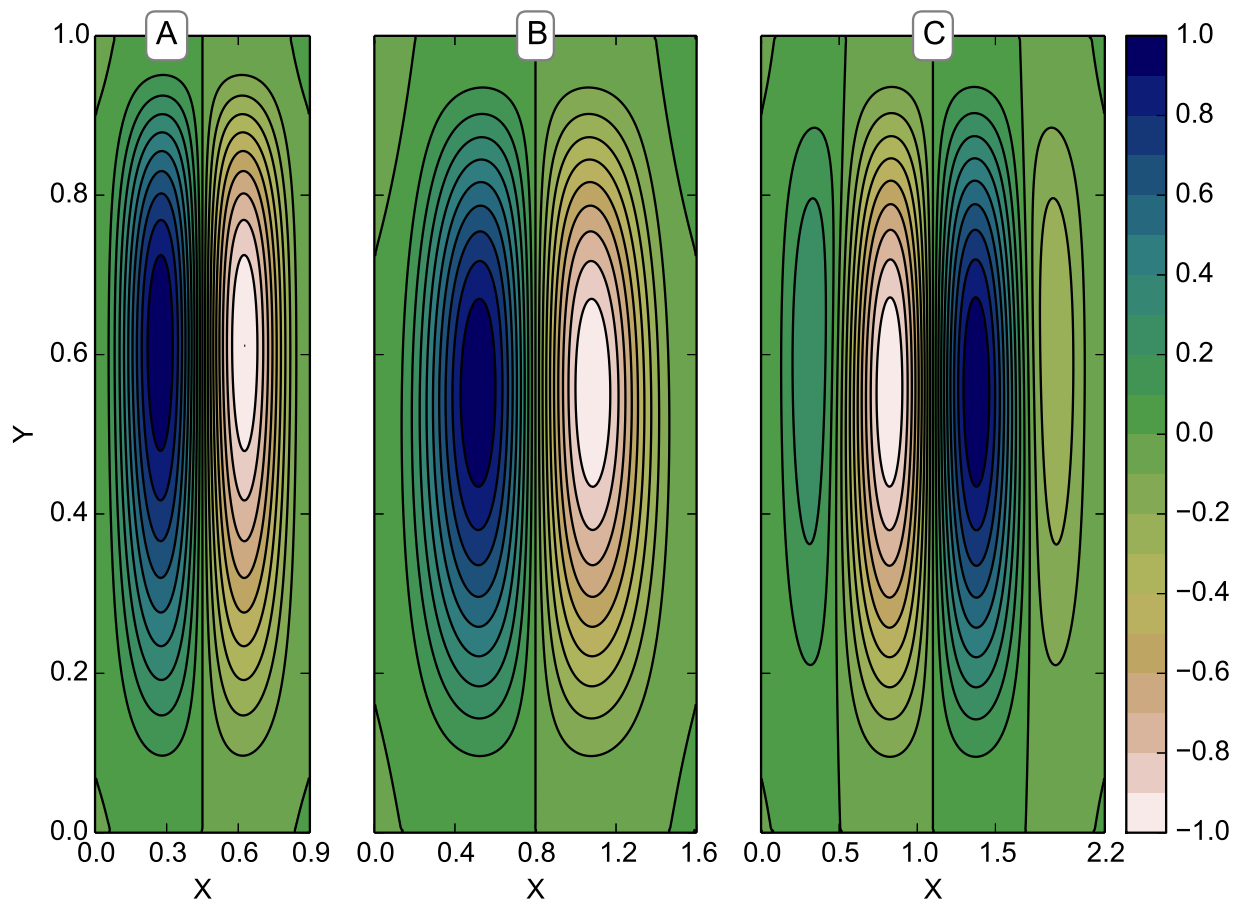

Figure 9. Stream function contour plots of the eigenfunction of mode 2 for $L=0.900,1.600,2.200$. This is the most unstable mode for the second value of $L$.

a subcritical bifurcation, no matter what boundary conditions are applied to the lateral walls. The numerical simulations presented in (Wu et al. 2013b) also showed that the structure of the flow pattern changes appreciably along the bifurcation diagram when a supercritical bifurcation takes place. The conclusion is that the supercritical behavior is the result of the competition between various modes. This competition appears because the bifurcation path of modes of the same symmetry class avoid crossing, compelling the path of one mode to change direction. We see in figure 6 that this results in the closing up of modes 1 and 2 after their first crossing. In this way both modes become unstable almost simultaneously when incrementing the stability parameter.

\section{Conclusions}

In this paper we have performed the linear stability analysis of the $2 \mathrm{D}$ electroconvective flow in a rectangular enclosure with no-slip conditions for velocity on all the boundaries. We have applied a perturbation technique. The mathematical procedure results in the definition of an eigenvalue problem, where the smallest eigenvalue gives the linear stability criterion. The finite element method is used to build a matrix eigenvalue problem that approximates the eigenvalues of the continuum problem. We first validate the overall procedure with the free walls case. After that, we have examined the structure of the eigenvalues and eigenfunctions for the rigid walls problem. The results are compared with the linear stability criterion obtained by the authors in a previous paper using a finite volume method to directly solve all governing equations. The agreement turns 

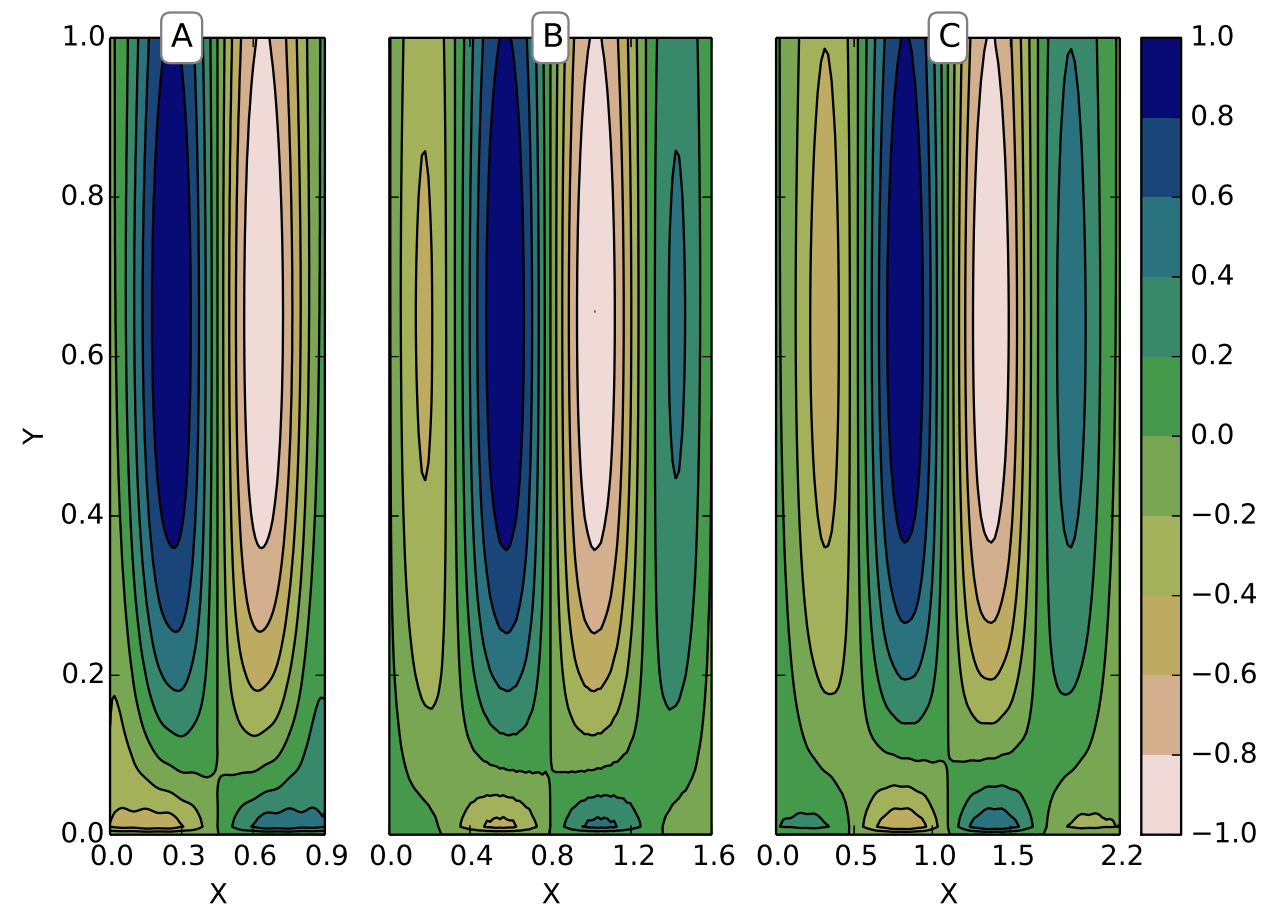

FiguRE 10. Contour plots of the charge density perturbation of mode 1 for $L=0.614,1.600,2.200$.

out to be excellent. The linear stability threshold approaches that of the infinite system when the width becomes very large. Also, when the width increases there is a repulsion between the modes with the same parity. This structure is a consequence of the different symmetry properties of the modes and is similar to what is obtained in Rayleigh-Bénard and Marangoni problems in enclosed boundaries.

\section{Acknowledgments}

This work has been carried out with financial support from the Spanish Ministerio de Ciencia y Tecnología (MCYT) under Research Project No. FIS2011-25161, and Junta de Andalucía under research projects P10-FQM-5735 and P09-FQM-4584. It was also partially funded by the French Government program Investissements d'Avenir (LABEX INTERACTIFS, reference ANR-11-LABX-0017-01) and by a grant from the french district PoitouCharentes.

\section{REFERENCES}

Atten, P \& Lacroix, J C 1979 Non-linear hydrodynamic stability of liquids subjected to unipolar injection. Journal de M\{é\} canique 18 (3), 469-510.

Atten, P, Lacroix, J C \& Malraison, B 1980 Chaotic motion in a coulomb force driven instability: Large aspect ratio experiments. Physics Letters A 79 (4), 255-258.

Atten, P \& Moreau, R 1972 Stabilité électrohydrodynamique des liquides isolants soumis à une injection unipolaire. Journal de Mécanique 11 (3), 471-520.

Boffi, D 2010 Finite element approximation of eigenvalue problems. Acta Numerica 19, 1-120. 

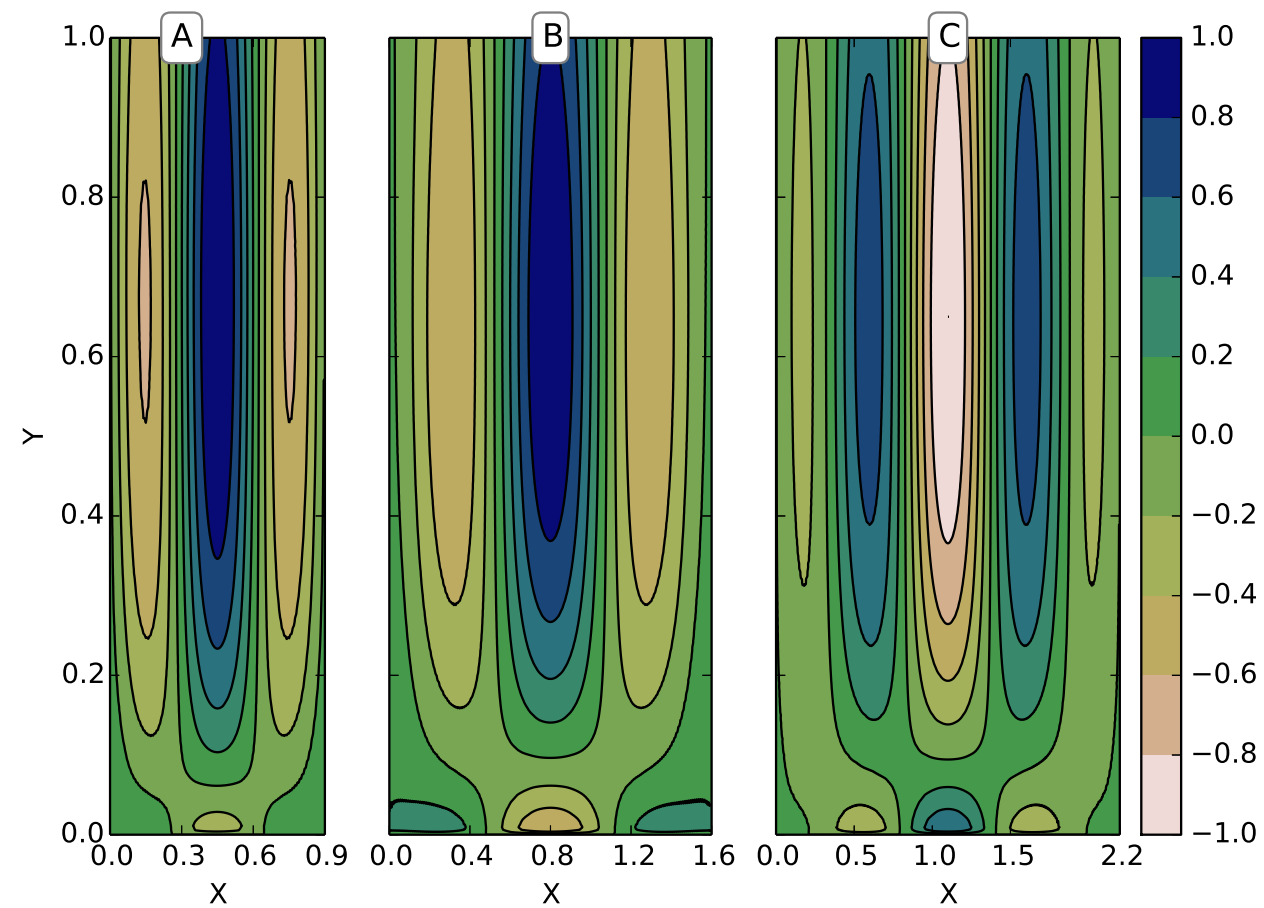

FiguRE 11. Contour plots of the charge density perturbation of mode 2 for $L=0.614,1.600,2.200$.

Castellanos, A 1991 Coulomb-driven Convection in Electrohydrodynamics. IEEE Transactions on Electrical Insulation 26 (6), 1201-1215.

Castellanos, A, ed. 1998 Electrohydrodynamics. Springer-Verlag.

Castellanos, A, Atten, P \& PÉrez, A T 1987 Finite amplitude electroconvection in liquids in the case of weak unipolar injections. PCH, PhysicoChemical Hydrodynamics 9 (3/4), 443-452.

Castellanos, Antonio, Ramos, Antonio, González, A, Green, N G \& Morgan, Hywel 2003 Electrohydrodynamics and dielectrophoresis in microsystems: scaling laws. Journal of Physics D: Applied Physics 36 (20), 2584-2597.

Chicón, R., Castellanos, A. \& Martin, E. 1997 Numerical modelling of Coulomb-driven convection in insulating liquids. Journal of Fluid Mechanics 344, 43-66.

Cliffe, K.A \& Winters, K.H 1986 The use of symmetry in bifurcation calculations and its application to the Bénard problem. Journal of Computational Physics 67 (2), 310-326.

Cliffe, K A, Garratt, T J \& Spence, A 1994 Eigenvalues of block matrices arising from problems in fluid mechanics. SIAM Journal on Matrix Analysis and Applications 15 (4), $1310-1318$.

Crowley, J M 1986 Fundamentals of Applied Electrostatics. Wiley.

Darabi, J, Ohadi, M \& DeVoe, D L 2001 An electrohydrodynamic polarization micropump for electronic cooling applications. Journal of Microelectromechanical Systems 10 (1), 98106.

Eds, Wells, Logg, Mardal, Wells, Logg, A, Mardal, K A \& Wells, G N 2010 Automated Solution of Differential Equations by the Finite Element Method. Springer.

Felici, N. J. 1969 Phénomenes hydro et aérodynamiques dans la conduction des diélectriques fluides. Revue General d'Electrostatique 78, 717-734.

Hernandez, Vicente, Roman, Jose E \& Vidal, Vicente 2005 \{SLEPc\}: A Scalable and 
Flexible Toolkit for the Solution of Eigenvalue Problems. ACM Transactions on Mathematical Software 31 (3), 351-362.

J C Lacroix, P Atten \& Hopfinger, E J 1975 Electroconvection in a Dielectric Liquid Layer Subjected to Unipolar Injection. Journal of Fluid Mechanics 69, 539-563.

JalaAl, M, Khorshidi, B \& Esmaeilzadeh, E 2013 Electrohydrodynamic (EHD) mixing of two miscible dielectric liquids. Chemical Engineering Journal 219 (0), 118-123.

Jones, T.B. 1979 Electrohydrodynamically Enhanced Heat Transfer in LiquidsA Review. Advances in Heat Transfer 14, 107-148.

LogG, A \& Wells, G N 2010 DOLFIN: Automated finite element computing. ACM Transactions on Mathematical Software 37 (2), 20:1--20:28.

Malraison, B \& Atten, P 1982 Chaotic behavior of Instability Due to Unipolar Ion Injection in a Dielectric Liquid. Physical Review Letters 49 (10), 723 - 727.

McCluskey, F M J \& Atten, P 1988 Modifications to the wake of a wire across Poiseuille flow due to a unipolar space charge. Journal of Fluid Mechanics 197, 81-104.

Melcher, J. R. 1981 Continuum Electromechanics. MIT Press.

Pearson, Matthew R \& Seyed-Yagoobi, J 2009 Advances in electrohydrodynamic conduction pumping. IEEE Transactions on Dielectrics and Electrical Insulation 16 (2), 424-434.

PÉrez, A T \& Castellanos, A 1989 Role of charge diffusion in finite-amplitude electroconvection. Physical Review A 40 (10), 5844-5855.

Ryu, J C, PARK, H J, PARK, J K \& KAng, K H 2010 New electrohydrodynamic flow caused by the Onsager effect. Physical Review Letters 104, 104502.

Schneider, J M \& Watson, P K 1970 Electrohydrodyamic stability of Space-Charge-Limited Currents in Dielectric Liquids I. Theoretical study. The Physics of Fluids 13 (8), 19481954.

SEyed-YAgoobi, J 2005 Electrohydrodynamic pumping of dielectric liquids. Journal of Electrostatics 63 (6), 861-869.

SEYED-YAGOOBI, J \& BRYAN, J 1999 Enhancement of heat transfer and mass transport in single-phase and two-phase flows with electrohydrodynamics. Advances in Heat Transfer 33, 99-186.

Traoré, Ph \& Louste, C. 2011 Numerical analysis of the effect of an EHD actuator on the flow past a square cylinder. In Dielectric Liquids (ICDL), 2011 IEEE International Conference on, pp. 1-4.

Traore, P \& Perez, A T 2012 Two-dimensional numerical analysis of electroconvection in a dielectric liquid subjected to strong unipolar injection. Physics of Fluids 24 (3), 037102.

VÁzquez, P A \& CAstellanos, A 2013 Numerical simulation of EHD flows using Discontinuous Galerkin Finite Element methods. Computers \& Fluids 84 (0), 270-278.

VÁzquez, P A, Georghiou, G E \& Castellanos, a 2006 Characterization of injection instabilities in electrohydrodynamics by numerical modelling: comparison of particle in cell and flux corrected transport methods for electroconvection between two plates. Journal of Physics D: Applied Physics 39 (13), 2754-2763.

VÁzquez, P A, Georghiou, G E \& Castellanos, A 2008 Numerical analysis of the stability of the electrohydrodynamic (EHD) electroconvection between two plates. Journal of Physics D: Applied Physics 41 (17), 175303:1 — 175303:10.

VAn DE Vooren, A.I. I \& DuKstra, H.A. A 1989 A finite element stability analysis for the Marangoni problem in a rectangular container with rigid sidewalls. Computers \& Fluids 17 (3), 467-485.

Winters, K H, Lesser, T H \& Cliffe, K A 1988 The onset of convection in a finite container due to surface tension and buoyancy. Physica D: Nonlinear Phenomena 29 (3), 387-401.

Wong, P K, Wang, T H, Deval, J H \& Ho, M C 2004 Electrokinetics in micro devices for biotechnology applications. EEE/ASME Trans. Mechatron. 9, 366-376.

Wu, Jian, Traoré, P \& Louste, C 2013a An efficient finite volume method for electric fieldspace charge coupled problems. Journal of Electrostatics 71 (3), 319-325.

Wu, J, Traoré, P, VÁzquez, P A \& PÉrez, A T $2013 b$ Onset of convection in a finite two-dimensional container due to unipolar injection of ions. Physical Review E 88 (5), 053018. 